for all patients from our centre up to December 2016, including those receiving treatment on a named patient basis. Data was available for $150 / 153$ patients started on pirfenidone and $56 / 57$ on nintedanib. Table 1 compares patients who were dispensed treatment but never took it, patients who stopped after the first prescription, and those continuing treatment. 8 patients consented to treatment but never took the drug; they were older and had less advanced disease. 39 patients stopped treatment after the first prescription; they had slightly more advanced disease, and had a longer interval between drug delivery and the first nurse (60.7 SD 57 days vs. 45.4 SD 30; $(\mathrm{p}=0.032)$, and more often had a shared care arrangement, but did not have a lower BMI. Photosensitivity for pirfenidone and diarrhoea for nintedanib were usually managed by treatment and were uncommon reasons for stopping antifibrotic therapy, which was more often related to upper GI intolerance and disease progression.

Conclusion Patients stopping antifibrotic treatment early cannot be identified from baseline data but can be reduced by intensive nursing support. Prescribing centre based ILD-CNS's should be responsible for early treatment tolerability and aim to see patients monthly until established on treatment. Uncertain delays between prescription and drug delivery make this more difficult.

\begin{tabular}{|c|c|c|c|}
\hline & \multicolumn{3}{|c|}{ Prescription dispensed } \\
\hline & $\begin{array}{l}\text { Once and never } \\
\text { taken }\end{array}$ & $\begin{array}{l}\text { Once and not } \\
\text { repeated }\end{array}$ & continued \\
\hline Number & 8 & 39 & 159 \\
\hline FVC\% predicted (SD) & $89.5(27)$ & $69.3(16)$ & $74.8(14)$ ** \\
\hline Composite physiology & $36.4(16)$ & 56.0() & $48.9(10)$ \\
\hline index & & & $* * *$ \\
\hline Shared care\% & & 38 & 20 ** \\
\hline Age & $78.0(6)$ & $72.5(7)$ & $70.4(9)$ * \\
\hline BMI & $30.0(2)$ & $28.5(6)$ & $29.2(5)$ \\
\hline
\end{tabular}

\section{P157 CAN BASELINE PHYSIOLOGICAL TESTS HELP PREDICT THE OUTCOME OF HYPOXIC CHALLENGE TESTING (HCT) IN INTERSTITIAL LUNG DISEASE (ILD)?}

${ }^{1} \mathrm{SL}$ Barratt, ${ }^{2} \mathrm{~J}$ Shaw, ${ }^{1} \mathrm{R}$ Jones, ${ }^{1} \mathrm{H}$ Adamali, ${ }^{3} \mathrm{C}$ Cliff, ${ }^{2} \mathrm{~N}$ Clayton, ${ }^{3} \mathrm{~N}$ Mustfa, ${ }^{3} \mathrm{H}$ Stone, ${ }^{2} \mathrm{~N}$ Chaudhuri. ${ }^{1}$ Bristol interstitial Lung Disease Service, Bristol, UK; ${ }^{2}$ University Hospital of South Manchester, Manchester, UK; ${ }^{3}$ Royal Stoke University Hospital, Stoke, UK

\subsection{6/thoraxjnl-2017-210983.299}

Introduction The British Thoracic Society guidelines suggest that pre-flight risk assessment should be considered in all individuals with Interstitial Lung Disease (ILD) intending to undertake commercial air travel. Hypoxic challenge testing (HCT) can be used to aid decisions about the need for inflight oxygen but there is a lack of evidence as to which patient variables might predict the outcome of HCT to guide referral for assessment.

Objective To investigate variables that might predict a hypoxaemic response to HCT in patients with ILD.

Methods A multi-centre retrospective analysis of all ILD patients attending for HCT at three tertiary care ILD referral centres between January 2010 and March 2017 was undertaken. The outcome of HCT was correlated to baseline demographic data, oxygen saturations (SpO2), capillary ear lobe $\mathrm{PaO} 2$, pulmonary function testing, 6MWT and GAP index, performed within 6 months of the HCT. Groups were compared using unpaired t-test with Welch's correction, unless otherwise stated $\quad(\mathrm{p}<0.05$ was considered statistically significant).

Results A total of 106 ILD patients (61 of whom (58\%) had IPF) underwent HCT. Of these, 54 (51\%) patients (of whom $30(49 \%)$ had IPF) failed HCT and were recommended supplemental in-flight oxygen. ILD patients who failed HCT had significantly lower resting SpO2, FEV1, FVC and TLCO $\%$ predicted, but higher GAP index (Table 1). In addition to these variables, the IPF subgroup failing HCT also had significantly lower minimum SpO2 during 6MWT.

Conclusions To our knowledge this is the largest retrospective study exploring predictors of HCT outcomes in ILD. Several baseline physiological parameters are significantly different between those ILD patients requiring in-flight oxygen based on HCT, and those who do not, including in a well-defined subgroup of IPF patients. Work is underway to establish a risk model to guide clinician decisions regarding the need for HCT in ILD.

\section{P158 PULMONARY VASCULAR DISEASE MARKERS PREDICT DEATH IN INTERSTITIAL LUNG DISEASE PATIENTS PROVEN NOT TO HAVE PULMONARY HYPERTENSION AT RIGHT HEART CATHETER}

${ }^{1}$ SRB Bax, ${ }^{1} \mathrm{C}$ Breedy, ${ }^{1} \mathrm{~K}$ Dimopoulos, ${ }^{1} \mathrm{~A}$ Kempny, ${ }^{1} \mathrm{~A}$ Devaraj, ${ }^{2} \mathrm{~S}$ Walsh, ${ }^{1} \mathrm{~J}$ Joseph, ${ }^{3} \mathrm{~S}$ Nair, ${ }^{1} \mathrm{M}$ Kokosi, ${ }^{4} \mathrm{G}$ Kier, ${ }^{1} \mathrm{C}$ Harries, ${ }^{1} \mathrm{~V}$ Kouranos, ${ }^{1} \mathrm{C}$ McCabe, ${ }^{1} \mathrm{~W}$ Li, ${ }^{5} \mathrm{M}$ Wilde, ${ }^{1} \mathrm{AU}$ Wells, ${ }^{1}$ LC Price*, 'SJ Wort*. ${ }^{1}$ Royal Brompton Hospital, London, UK; ${ }^{2}$ Kings College Hospital Foundation Trust, London, UK; ${ }^{3}$ Guy's and St Thomas' NHS Foundation Trust, London, UK; ${ }^{4}$ PrincessAlexandra Hospital, Brisbane, Australia; ${ }^{5}$ Surrey and Sussex NHS Trust, London, UK; *Joint final author

\subsection{6/thoraxjnl-2017-210983.300}

Introduction Patients with interstitial lung disease (ILD) often have signs of pulmonary hypertension $(\mathrm{PH})$ when assessed non-invasively. The presence of pulmonary hypertension $(\mathrm{PH})$ at right heart catheter (RHC) is a negative prognostic factor; however, the impact of elevated pulmonary vascular biomarkers in the absence of $\mathrm{PH}$ is poorly understood. We hypothesised that pulmonary vascular disease biomarkers would predict mortality in patients without PH at RHC.

Methods Demographics, ILD subtype, PFTs, echocardiogram, and CTs were reviewed in consecutive patients undergoing right heart catheterisation (RHC) for suspected ILD-PH. Patients with a mean pulmonary arterial pressure (mPAP) $<25 \mathrm{mmHg}$ at RHC were studied. Predictors of prognosis were evaluated in their ability to predict mortality using Cox proportional hazard analysis. 